\title{
Programmatically Identifying Cognitive Biases Present in Software Development
}

\author{
Amanda E. Kraft ${ }^{\ddagger \dagger}$, Matthew Widjaja ${ }^{\ddagger \dagger *}$, Trevor M. Sands ${ }^{\ddagger}$, Brad J. Galego
}

\begin{abstract}
Mitigating bias in Al-enabled systems is a topic of great concern within the research community. While efforts are underway to increase model interpretability and de-bias datasets, little attention has been given to identifying biases that are introduced by developers as part of the software engineering process. To address this, we began developing an approach to identify a subset of cognitive biases that may be present in development artifacts: anchoring bias, availability bias, confirmation bias, and hyperbolic discounting. We developed multiple natural language processing (NLP) models to identify and classify the presence of bias in text originating from software development artifacts.
\end{abstract}

Index Terms - cognitive bias, software engineering, natural language processing

\section{Introduction}

Artificial intelligence (AI) and machine learning (ML) -based systems are increasingly supporting decision-making, reasoning, and evaluation of dynamic environments in objective manners. As AI-enabled systems are finding increasing use across domains and industries, there is concern that the objectivity of such systems may be negatively impacted by biases introduced by the developers either in the design of the system or in the training data itself. While efforts are underway to make AI/ML systems more interpretable and debias datasets, little research is directed at human-centric cognitive biases that developers unintentionally introduce as a part of the software engineering (SE) process. As a result, ensuring unbiased and transparent algorithmic decisionmaking is a complex challenge and has wide-ranging implications for the future use of $\mathrm{AI}$ in society.

Cognitive biases are systematic deviations from rationality in judgment, reasoning, evaluation, or other cognitive processes. For the myriad of cognitive biases described in literature ${ }^{1}$, approximately 40 have been investigated in the SE domain ${ }^{2}$. We selected four of the most commonly reported cognitive biases in software engineering:

- Anchoring Bias: Tendency to rely too heavily on preexisting or first information found when making a quantitative judgment ${ }^{2}$.

$\dagger$ These authors contributed equally.

* Lockheed Martin Advanced Technology Laboratories

* Corresponding author: matthew.widjaja@lmco.com

Copyright $(2021$ Amanda E. Kraft et al. This is an open-access article distributed under the terms of the Creative Commons Attribution License, which permits unrestricted use, distribution, and reproduction in any medium, provided the original author and source are credited.
- Availability Bias: Tendency to overestimate the likelihood of events based on the ease of which examples come to $\operatorname{mind}^{3}$.

- Confirmation Bias: Tendency to search for and focus on information that confirms one's preconception(s) while ignoring or rejecting sources that challenge it ${ }^{4}$.

- Hyperbolic Discounting: Tendency to prefer immediate payoffs over larger rewards at a later point ${ }^{2}$.

These biases may be influenced by self-generated factors (e.g., past development experience), or externally generated factors (e.g., system documentation $)^{5}$. A tool to detect biases in software must be capable of assessing multiple sources of information about the system, including commit messages, comments, in-source docstrings, external technical documentation, and diagrams. This study takes the first steps toward this objective by identifying cognitive biases in software commit messages and comments from previously completed projects.

The remainder of this paper is organized into three sections: research methods, results and discussion, and conclusions and implications for future work in this space.

\section{Research Methods}

In this section we discuss how data was initially gathered and curated prior to annotation, how manual annotation was performed using Prodigy, the process for reviewing and finalizing the consensus for data labels, and finally, the approach for developing machine learning classifier models applied to the annotated data to determine whether bias is present in a given sample.

\section{Data Curation}

To address the lack of research identifying cognitive biases in software artifacts developed as part of a naturally occurring development process, we collated data from two internally developed codebases. The first project ("Project A") was selected to represent the whole software engineering process for AI/MLenabled systems (i.e., data management to feature extraction to model training and evaluation). The second project ("Project B") is similar in structure to the first, but the software artifacts gathered include only the latter half of the development cycle (i.e., feature extraction to model training and evaluation). The content from both codebases were collated into datasets based on the source of the development artifacts: commit messages, in-source code comments, and documentation strings (docstrings). Given the time limitations for this effort, we prioritized annotation of commit 


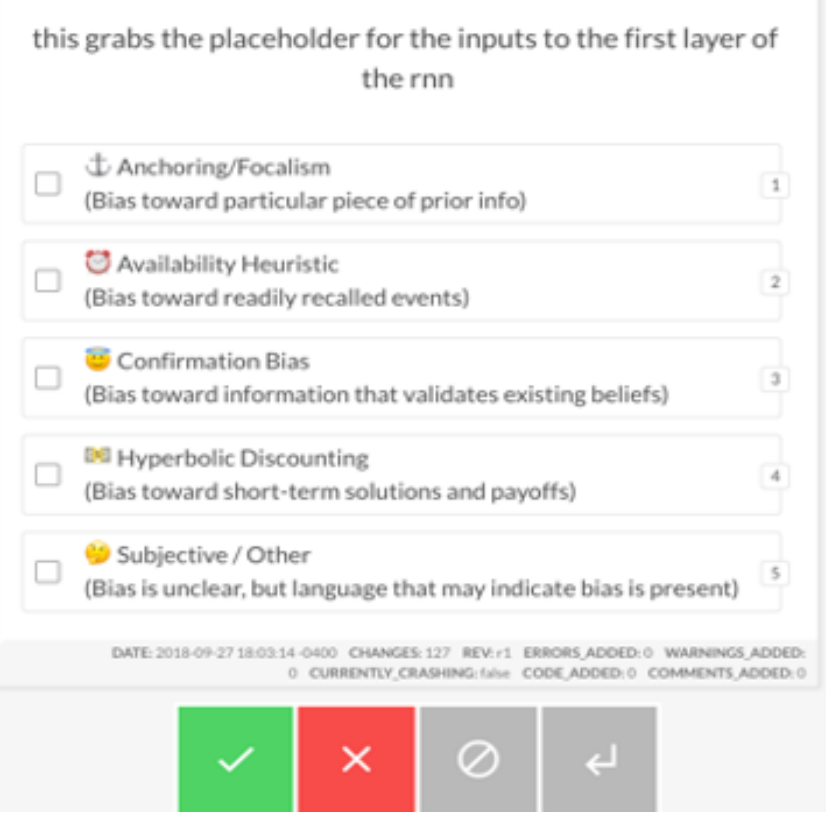

Fig. 1: Example view of a comment in reviewer mode. The reviewer has three options: (1) accept via the green checkmark if bias is detected, (2) reject via the red $X$ if no bias detected, and (3) ignore via the grey stop icon if the entry contains no intelligible content.

messages for all datasets, while comments and docstrings were annotated for the second of the two internal projects.

Further, we identified an open-source dataset, Code Smell ${ }^{6}$, to validate models trained on the content from the internal projects. This dataset contains commit messages extracted from refactoring activities across the software lifecycle for various open-source projects.

For all datasets, python scripts were developed to programmatically extract and format the text content from the artifacts. Specifically, the following operations were performed: commit message content had whitespace trimmed and artifact identifiers removed; comments spanning multiple lines were combined into a single entry; executable segments of code were removed; entries with non-ASCII characters were removed; references to individual names, collaboration teams, applications, and projects were redacted and replaced with an identifier string (e.g., "NAME1").

\section{Bias Annotation: Prodigy Setup}

The processed text data was then annotated in Prodigy to produce a structured JSON document. Prodigy is a commercially licensed software tool for collaborative data annotation. A custom annotation recipe was developed to define the four biases described above as the possible labels; an additional label option, "Subjective/Other" was included to provide reviewers a chance to flag entries containing a form of bias other than the available options. Figure 1 provides an example of what individual reviewers see when annotating a given dataset using this custom recipe. For each entry, the reviewer must decide whether an entry is valid, and if so, if the language indicates that the author may have introduced bias into the system. When reviewers determined an entry contains bias, they selected one or more labels and pressed "accept"; otherwise, the reviewer pressed "reject" to indicate no language indicating bias was present.

\section{Bias Annotation: Manual Annotation}

A total of six reviewers were engaged in this project for the bias annotation process. All reviewers have at least two years of programming experience and are between the ages of 18-40. Two reviewers are female and four are male. Two reviewers are Asian, while the other four are White. Three of the six reviewers had some degree of involvement in developing the software for two of the internal projects discussed in this paper. Further, one of these reviewers was the software lead on Project B. To minimize personal biases when reviewing the development artifacts, all entries are anonymized and annotated in non-chronological order.

An annotation guide for classifying open-ended text entries was developed for reviewers to remain consistent. The guide provides examples of several biased commit messages such as:

- Anchoring Bias

- $\quad$ "Extended module to allow a more traditional approach to interface engineering"

- "Applying back-changes from my original fix patch"

- $\quad$ "Correct the temperature unit - assumes anything under 45 is $\mathrm{C}^{\prime \prime}$

- Availability Heuristic

- "Renamed method to more sensible wording"

- "Tighter coupling of variable names with other modules"

- Confirmation Bias

- $\quad$ "The use of $[\mathrm{X}]$ rather than [Y] allows each module to reuse the same functionality without having to extend a base class"

_ "We're now a bit smarter about the size of tables that we create by default, which was the root of the prior problems"

- Hyperbolic Discounting

- $\quad$ "Throwing out the Key and Value classes for now to reduce the overall complexity"

- $\quad$ "Modified function to account for type errors. Will likely have to recreate the $\mathrm{db}$ every time, unless other solutions come up"

_ "Module incorporated but fails"

- "Quick and dirty method to add features"

- Subjective/Other

- $\quad$ "I was too over-zealous with removing a module"

- $\quad$ "Duplicate code is my nemesis..."

The guide reminds reviewers that they are to label if the language indicates the author may have introduced bias into the system, not if the language indicates the author may be addressing bias previously introduced. The guide further advises the reviewer to flag entries as invalid if they should be excluded from the training or testing datasets; the exclusion criteria include blank messages, machine-generated messages (e.g., automated branch merging messages), messages only containing an artifact or issue identifier, and "TODO" or "FIXME" comments with no accompanying description. Reviewers were also encouraged to accept samples that may be borderline cases, as a group consensus would decide final classification labels. 


\section{Bias Annotation: Finalizing Bias Labels}

After all reviewers submitted their final annotations for a dataset, one reviewer was selected to finalize the labels to be used for training and testing models. For consistency, the same reviewer was selected to finalize labels on all datasets. The review process itself was facilitated by Prodigy, which offers a built-in review recipe, allowing a user to specify the annotation databases to use. With this recipe, Prodigy extracts all instances where an entry was marked as "accepted" or "ignored" by at least one reviewer. These are compiled and displayed similar to the initial review, noting which review session(s) indicated which label(s).

In the final review, a "best fit" label was selected, rather than accepting multiple labels for a single entry as allowed in the initial review stage. This decision was implemented in order to provide non-overlapping classification boundaries for model training and testing. The final reviewer followed a set of guidelines for determining best fit labels, such as cross-referencing the annotation guide or identifying the word or phrase that may have triggered the response when multiple reviewers selected different biases for a single message.

If the final reviewer thought the best fit label was ambiguous or if the label selected was only reported by themselves during the initial review process, the message was logged for additional review. These flagged messages were compiled in an Excel workbook along with the selected answer (first-degree label), the next best answer (second-degree label), and the labels marked by the initial review sessions. The workbook was sent to at least two individuals to respond to these entries, indicating their judgement of whether the first or second degree label was the best fit or if another label option may have been overlooked. Scoring of their responses was automated using the following rules: (1) if both agreed with the first degree label, it was kept; (2) if both agreed with the second degree label, the final label was switched; (3) if the first degree label was not "reject" and one accepted while the other rejected, the first degree label was kept. On the rare occasion when none of these conditions were met, the final reviewer decided the label selection based on the feedback.

The results of the final review (i.e., entries labeled as biased) were merged with the source dataset (i.e., non-biased entries) to comprise the training and testing datasets for modeling.

\section{Models}

To determine whether a tool can classify software artifacts as containing indicators of bias, we developed text classification models using spaCy. Binary and multi-class models were considered, where binary models were concerned with identifying the presence or absence of biased language and multi-class models concerned with identifying the type of bias present (if one is present at all). Anticipating that the class distributions would be highly imbalanced towards not containing bias, we implemented down sampling by taking the mean of the quantity of data present across each label type to improve model training. This method was randomized, with ten models trained on different data distributions.

Focusing on the ability of the trained models to perform on different codebases, we prioritized evaluating the models independently trained on the two internal commit datasets and applied each to the Code Smell dataset (i.e., as a test dataset). As a secondary task, we then combined the internal commits in a single training set and applied them to Code Smell. Additionally, to determine if commit messages can predict bias in comments,

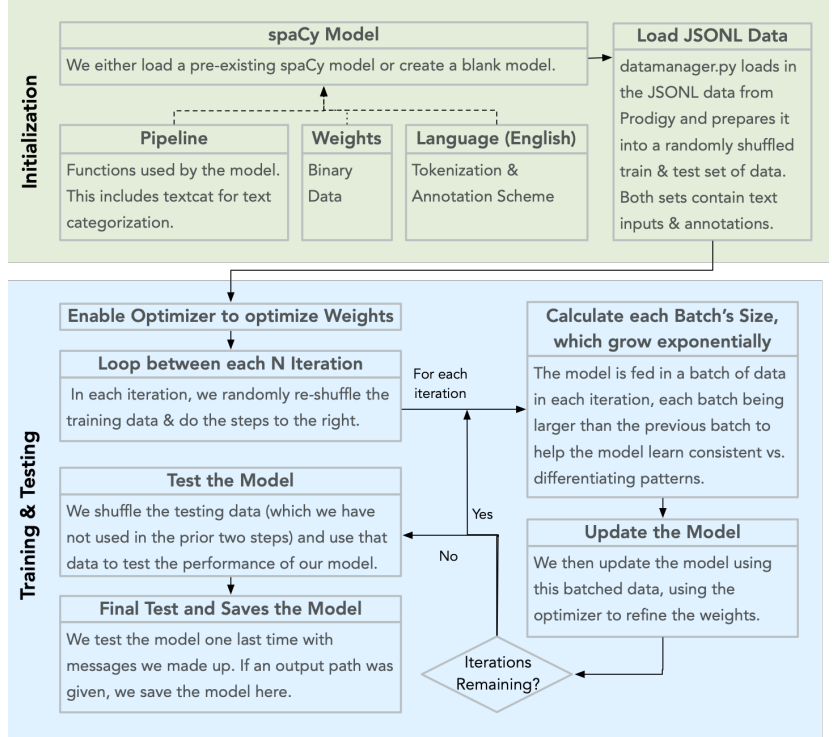

Fig. 2: Overview of the spaCy NLP modeling workflow, broken up into initialization (green) and execution (blue) task phases.

we trained a model on the internal commits and tested against comments for the same project. Finally, we evaluate the combined internal dataset against Code Smell.

We ran each model three times, each time using a different split of the dataset. This modeling process is illustrated in Figure 2. For each model, we report the mean F1 score and standard deviation across runs. We swept across three model hyperparameters during training:

1) The maximum number of samples used to train: This mitigates the impact of label imbalance, by limiting the total number of entries from each category before training the model. The considered caps included:

1. The quantity of entries from all biases.

2. The mean of the quantity of data from each category, including data which was not biased.

3. The quantity of entries from the largest bias category.

4. No capacity, use all data.

2) Dropout: This is the percentage of connections which are dropped from the neural network component of the ensemble learning and is used to prevent over-fitting. Typical sweep values are $20 \%, 40 \%$, and $60 \%$.

3) The size of the training batches and their compounding rate at each epoch: This determines how much data is passed to the trainer at each iteration from a minimum (batch start size) to a maximum (batch stop size) with a given rate of growth (compounding rate). For all models, the compounding rate was left at the spaCy recommended value of 1.001 .

\section{Results and Discussion}

In this section we discuss the results of data annotation and the classifier models. Statistics about the annotated data including the final label distributions and interrater reliability are presented. Model hyperparameters are presented and discussed with respect to their mean F1 scores and standard deviations. 


\begin{tabular}{|l|c|c|c|c|}
\hline Dataset & $\begin{array}{c}\text { Total } \\
\text { Items }\end{array}$ & $\begin{array}{c}\text { Duplicate } \\
\text { Items }\end{array}$ & $\begin{array}{c}\text { Final Item } \\
\text { Count }\end{array}$ & Reviewers \\
\hline $\begin{array}{l}\text { Code Smell } \\
\text { Commits }\end{array}$ & 471 & 30 & 441 & 5 \\
\hline $\begin{array}{l}\text { Project A } \\
\text { Commits }\end{array}$ & 1536 & 131 & 1405 & 6 \\
\hline $\begin{array}{l}\text { Project B } \\
\text { Commits }\end{array}$ & 238 & 11 & 227 & 5 \\
\hline $\begin{array}{l}\text { Project B } \\
\text { Comments }\end{array}$ & 469 & 0 & 469 & 5 \\
\hline $\begin{array}{l}\text { Project B } \\
\text { Docstrings }\end{array}$ & 181 & 0 & 181 & 5 \\
\hline
\end{tabular}

(i.e., "accept", "reject", or "ignore") is divided by the total number of possible pairs. For label reliability, we start with the high-level measure of all label options, including the empty label string that results from selection of "reject" or "ignore". We refer to this measure as annotation reliability, as it accounts for a combination of answer and label selection, though at the cost of instances of "reject" and "ignore" being indistinguishable. Given the expected imbalance of bias versus non-biased entries, we also provide an average of the reliability scores for the subset in which at least one bias label is selected. We refer to this measure as sub-annotation

TABLE 1: Overview of the five datasets, including: (1) counts of original entries, (2) duplicate entries, excluding first occurrence, (3) final entry count with duplicates removed, and (4) number of reviewers that annotated each dataset.

\begin{tabular}{|l|c|c|c|c|}
\hline Dataset & Answer & Annotation & $\begin{array}{c}\text { Sub } \\
\text { Annotation }\end{array}$ & Bias \\
\hline $\begin{array}{l}\text { Code Smell } \\
\text { Commits }\end{array}$ & $0.85 \pm 0.23$ & $0.83 \pm 0.28$ & $0.44 \pm 0.19$ & $0.22 \pm 0.35$ \\
\hline $\begin{array}{l}\text { Project A } \\
\text { Commits }\end{array}$ & $0.86 \pm 0.21$ & $0.87 \pm 0.24$ & $0.50 \pm 0.20$ & $0.39 \pm 0.40$ \\
\hline $\begin{array}{l}\text { Project B } \\
\text { Commits }\end{array}$ & $0.78 \pm 0.24$ & $0.89 \pm 0.24$ & $0.43 \pm 0.21$ & $0.35 \pm 0.38$ \\
\hline $\begin{array}{l}\text { Project B } \\
\text { Comments }\end{array}$ & $0.91 \pm 0.19$ & $0.92 \pm 0.20$ & $0.51 \pm 0.17$ & $0.43 \pm 0.48$ \\
\hline $\begin{array}{l}\text { Project B } \\
\text { Docstrings }\end{array}$ & $0.95 \pm 0.15$ & $0.94 \pm 0.16$ & $0.51 \pm 0.15$ & $0.42 \pm 0.49$ \\
\hline
\end{tabular}

TABLE 2: Interrater reliability across the annotated datasets as percentages, with a +/- standard deviation. "Answer" refers to the annotation response type (i.e., accept, reject, ignore). "Annotation" considers the specific bias label, where reject/ignore are empty strings. "Sub-Annotation" considers the subset of entries in which at least one reviewer selected a bias label. "Bias" compares only the bias labels selected by reviewers (i.e., reject/ignore responses are not considered). Reviewers typically agree on whether an entry is biased, but not on the bias type.

\section{Annotated Datasets}

An overview of the four datasets in terms of total number of items, number of duplicate entries, final number of items after accounting for duplicates, and number of reviewers to annotate is provided in Table 1.

To quantify variance in interpretation of bias presentation in software commit messages and comments, interrater reliability was computed based on percent agreement across reviewers. Percent agreement is computed as the number of matching pairs over the number of total possible pairs.

For answer reliability, the number of matching answer pairs

\begin{tabular}{|l|c|c|c|c|}
\hline Dataset & $\begin{array}{c}\text { Total } \\
\text { Items }\end{array}$ & $\begin{array}{c}\text { Rejected } \\
\text { (Not Biased) }\end{array}$ & $\begin{array}{c}\text { Accepted } \\
\text { (Biased) }\end{array}$ & $\begin{array}{c}\text { Ignored } \\
\text { (Excluded) }\end{array}$ \\
\hline $\begin{array}{l}\text { Code Smell } \\
\text { Commits }\end{array}$ & 441 & 389 & 51 & 1 \\
\hline $\begin{array}{l}\text { Project A } \\
\text { Commits }\end{array}$ & 1,405 & 1,154 & 162 & 89 \\
\hline $\begin{array}{l}\text { Project B } \\
\text { Commits }\end{array}$ & 227 & 140 & 26 & 61 \\
\hline $\begin{array}{l}\text { Project B } \\
\text { Comments }\end{array}$ & 469 & 430 & 27 & 12 \\
\hline $\begin{array}{l}\text { Project B } \\
\text { Docstrings }\end{array}$ & 181 & 174 & 7 & 0 \\
\hline
\end{tabular}

TABLE 3: Overview of the finalized annotations for each dataset. Entries labeled as "ignore" are excluded from the datasets for subsequent modeling.

reliability. Lastly, we compute a bias reliability measure in which we compare only the label options available when a reviewer "accepts" an entry as biased.

There were six reviewers for the Project A Commits dataset and five reviewers for all other datasets. Interrater reliability was computed across reviewer annotations and are summarized in Table 2. The distributions of bias labels for each dataset are represented in Figure 3. Overall, reliability measures ranged from 0.78 to 0.91 for answers, 0.83 to 0.92 for annotations, 0.43 to 0.51 for sub-annotations, and 0.22 to 0.43 for bias labels across the four datasets. An overview of the final annotation labels is provided in Table 3.

Given the nature of the data being annotated, we expected a significant amount of variance in how reviewers interpret commit messages and in-source comments, especially without additional context about the relevant code. This was confirmed with the interrater reliability for top-level answers averaging to $85 \%$ agreement, while reliability on bias type averaged to $35 \%$. However, we didn't expect the level of disagreement to be so high, especially when reviewing the label distributions by reviewer. For example, some reviewers used "Other" or selected multiple labels at a much greater rate than others. This may have resulted from the reviewers being unclear on what specific bias was present.

Further, the overall distribution of biased versus not biased entries by dataset supports that artifact types (e.g., comment, commit) are used differently. For example, both comments and docstrings tend to be more technical in nature, with comments typically reflecting procedural knowledge and docstrings describing the purpose, inputs, and outputs of a class or function. This is reflected by all three commit message datasets having approximately $12 \%$ of messages flagged as biased, while comments and docstrings only had $6 \%$ and $4 \%$ biased entries, respectively.

\section{Modeling}

Table 4 summarizes the results for each model, along with the best-performing hyperparameters as determined by a parameter sweep. The mean and standard deviation of F1 Scores are computed across three randomized train/test splits within the same dataset.

No models were trained using the dataset comprised of docstrings due to the extreme imbalance in labels (i.e., $<5 \%$ labeled as bias). The docstring dataset had a total of 7 (of 181) entries labeled as biased. This may be attributed to the inherit technical nature of docstrings, combined with the low quantity of docstrings collected during data curation.

The multi-label model $(\mathrm{F} 1=72.1 \%)$ did not meet expectations because it consistently predicted that no bias was typically present. This model was over-fit given that the biased entries were now split among four separate bias labels, increasing the level of imbalance. Though this finding may be due to insufficient training data availability, it's interesting to note that interrater reliability 

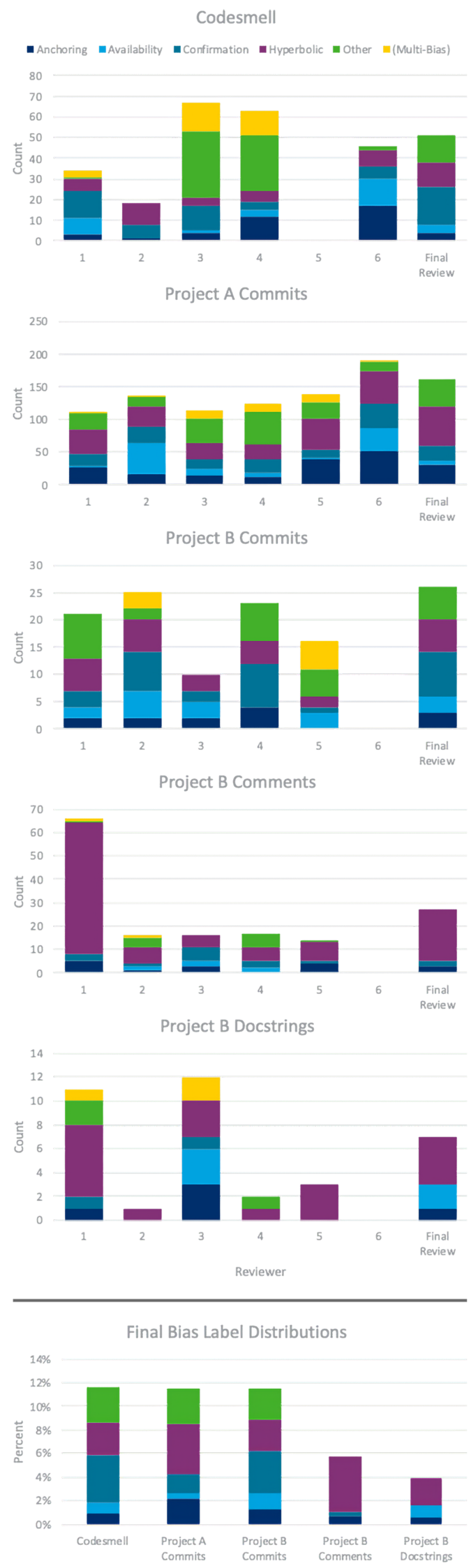

Fig. 3: Distribution of bias labels per dataset. The first five plots show the distribution of label counts by each reviewer and the finalized review process. The last plot shows the finalized distribution of labels as percentages to standardize visualization across all datasets. The final plot reflects the data used for modeling.

\begin{tabular}{|l|c|c|c|c|c|c|}
\hline Dataset & $\begin{array}{c}\text { Model } \\
\text { Type }\end{array}$ & $\begin{array}{c}\text { Max } \\
\text { Samples }\end{array}$ & $\begin{array}{c}\text { Drop } \\
\text { Rate }\end{array}$ & $\begin{array}{c}\text { Batch } \\
\text { Range }\end{array}$ & $\begin{array}{c}\text { Mean } \\
\text { F1 }\end{array}$ & $\begin{array}{c}\text { Std. } \\
\text { Dev. }\end{array}$ \\
\hline $\begin{array}{l}\text { Project A } \\
\text { Commits }\end{array}$ & Binary & 220 & $40 \%$ & $4-64$ & $81.2 \%$ & $2.6 \%$ \\
\hline $\begin{array}{l}\text { Project B } \\
\text { Commits }\end{array}$ & Binary & 28 & $20 \%$ & $8-64$ & $65.9 \%$ & $14.0 \%$ \\
\hline $\begin{array}{l}\text { Project A + } \\
\text { B Commits }\end{array}$ & Binary & 247 & $20 \%$ & $4-64$ & $79.0 \%$ & $5.1 \%$ \\
\hline $\begin{array}{l}\text { Project A + } \\
\text { B Commits }\end{array}$ & $\begin{array}{c}\text { Multi } \\
\text { Label }\end{array}$ & 188 & $20 \%$ & $8-32$ & $72.1 \%$ & $5.8 \%$ \\
\hline $\begin{array}{l}\text { Project B } \\
\text { Commits + } \\
\text { Comments }\end{array}$ & Binary & 104 & $40 \%$ & $8-32$ & $78.6 \%$ & $6.8 \%$ \\
\hline $\begin{array}{l}\text { All Internal } \\
\text { Data }\end{array}$ & Binary & 324 & $40 \%$ & $8-64$ & $82.3 \%$ & $3.9 \%$ \\
\hline
\end{tabular}

TABLE 4: Hyperparameters selected and corresponding results for each model. Model Type refers to whether the model predicted Bias vs. No Bias (Binary) or the particular bias types (Multi-Label). "Max Samples" refers to the maximum number of samples allowed for each bias category, to prevent over-fitting given data imbalance. "Drop Rate" and "Batch Range" are hyperparameters for the NLP model. "Mean F1" and "Std. Dev" refer to the model results across three randomized train/test splits within the same dataset.

follows a similar pattern when defining a specific bias label. The confusion matrices from each of the three instances of this model is Figure 4.

All binary classification models performed in parity with one another, with mean F1 scores ranging from $78.6 \%$ to $82.3 \%$. These models performed better than the multi-label models given less data imbalance between the binary categories (i.e., bias vs. no bias). The model trained on Project B Commits data was the only exception, which performed at $65.9 \%$, most likely due to the significantly smaller size of the training dataset.

The best performing model $(\mathrm{F} 1=82.3 \%)$ was trained using the largest dataset (i.e., the combined commit messages and comments for both Projects A and B) as a binary classification model. The confusion matrices of the three instances of this model is in Figure 5.

\section{Conclusions and Implications}

Through this project, two well-curated datasets were generated: one derived from the commit messages of Projects A and B and the other created by labeling an existing collection of code refactoring-related commit messages from various free and opensource software projects ${ }^{6}$. This data is valuable not only because it is the first of its kind, but also because it is representative of technical artifacts generated during the software development process.

The level of variability in bias annotations across reviewers emphasizes the difficulty in discerning whether a statement is biased without insight of the surrounding context. This is further exacerbated when it comes to identifying the type of bias. Furthermore, limiting reviewers to a single annotation per entry may alleviate the risk of reviewers selecting multiple labels when uncertain. Our interrater reliability inherently resulted in lower scores for multi-label annotations. For example, ['ANCHORING,HYPERBOLIC'] and ['HYPERBOLIC'] results in bias reliability of zero even though both reviewers thought hyperbolic discounting was present. The level of variation may also arise from individual differences in writing commit messages and comments; messages that are longer or enumerate each change made are more likely to elicit language suggestive of bias compared to 


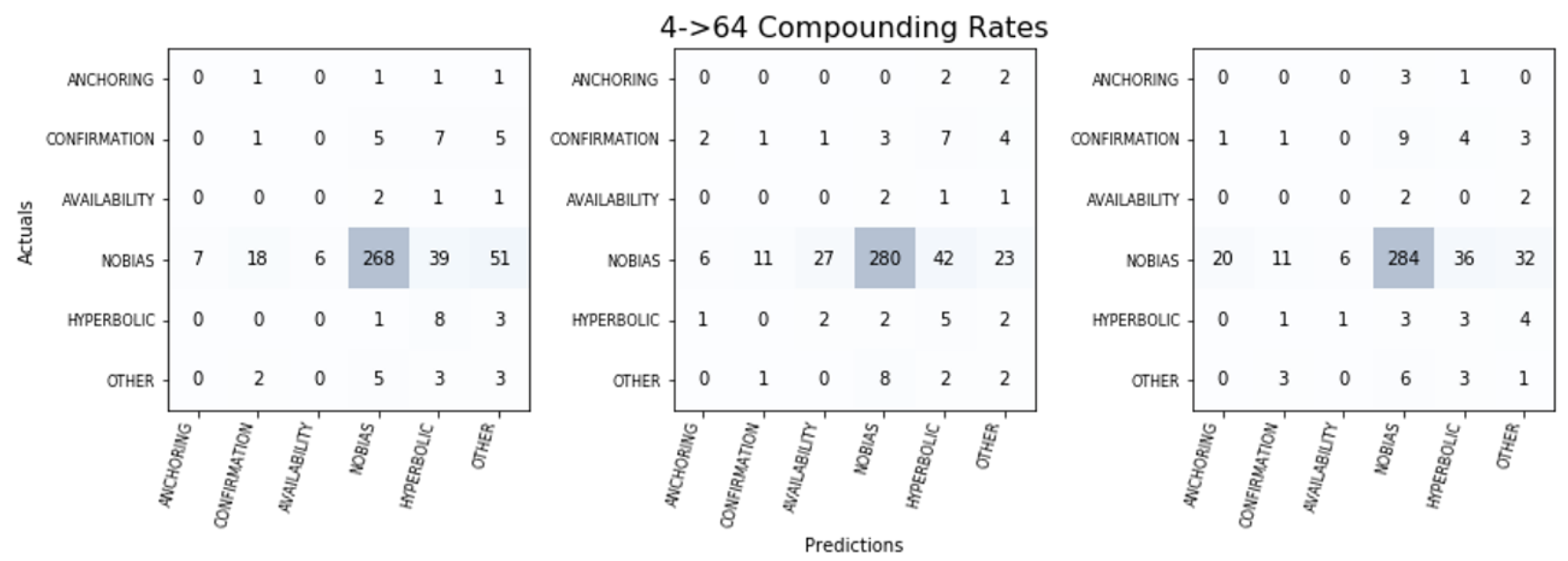

Fig. 4: Confusion matrices for the multi-label model on "Project A + B Commits". Each matrix is an instance of a model run on a randomized split of the data. All three exemplify over-fitting to the "no bias", attributable to data imbalance. This pattern also mirrors the low interrater reliability scores for specific bias labels.
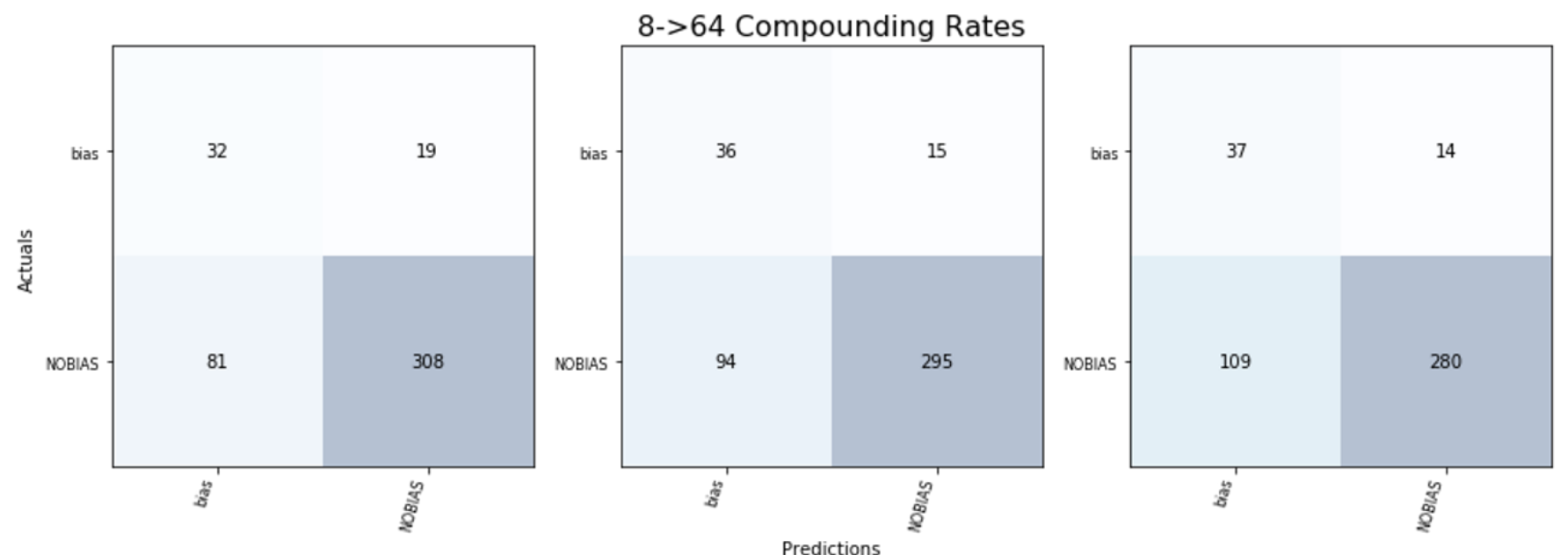

Fig. 5: Confusion matrices for the binary model on "All Internal Data". Each matrix is an instance of a model run on a randomized split of the data. This model performed the best overall, attributable to the larger dataset size and reduced data imbalance compared to the other models.

highly concise messages. Properly identifying bias in software artifacts may require consideration for informing software teams on message structuring for consistency and utility.

Possible follow-on efforts to this study will investigate further ways to improve multi-label modeling of bias. The multi-label model was over-trained due to the significant quantity of nonbiased data versus the other four categories of bias. This differs from the binary models, which had the advantage of being able to combine those four bias categories together, resulting in a more balanced dataset. Obtaining more data, specifically of entries which are biased, will likely improve model robustness for both the binary and multi-label models.

While data quantity remains an issue, we also note some disagreement in data labels, reflected in the interrater reliability (Table 2). It was not surprising to see the multi-label model struggle to select the correct bias label, as the annotators tended to disagree on which biases were present in specific data points. We had a process to select a single bias label for each entry from the pool of bias labels that the annotators independently selected. It is possible that our model actually agreed with one of the bias labels that an annotator voted on, but was rejected or changed during the final review label review. A follow-on effort to this study will better measure the multi-label model's performance against the pool of bias labels candidates, rather than the single entry selected during annotation review.

Future research efforts that can build on these results include the generation of datasets and models that consider the impact of individual words or short phrases on bias classification, application of a bias detection tool in tracing the source of a significant failure to the engineering process (as opposed to a particular line of code), and investigation of the impact of cognitive bias on code quality metrics. Additionally, larger datasets, especially ones containing in-code comments and document strings, are necessary to quantify the impact of cognitive biases on the quality of finished software systems. In the future, larger projects may require the development of post-mortem reports to identify which aspects of the research, design, and development cycles are most impactful to overall project success or failure. With such data available researchers can begin to answer the central question regarding the impact of individual biases from a holistic perspective. 


\section{Acknowledgements}

We thank Michael Krein, Lisa Baraniecki, and Owen Gift for their contributions to annotating the datasets used in this effort.

1. M. Delgado-Rodriguez and J. Llorca, Bias, Journal of Epidemiology \& Community Health, 58(8):635-641, 2004.

2. R. Mohanani, I. Salman, B. Turhan, P. Rodríguez and P. Ralph, Cognitive biases in software engineering: a systematic mapping study, IEEE Transactions on Software Engineering, 2018.

3. W. Stacy and J. MacMillan, Cognitive bias in software engineering, Communications of the ACM, 38(6):57-63, 1995.

4. G. Calikli and A. Bener, Empirical analysis of factors affecting confirmation bias levels of software engineers, Software Quality Journal, 23(4):695722, 2015

5. K. Mohan and R. Jain, Using traceability to mitigate cognitive biases in software development, Communications of the ACM, 51(9):110-114, 2008.

6. E. AlOmar, M. W. Mkaouer and A. Ouni, Can refactoring be selfaffirmed? an exploratory study on how developers document their refactoring activities in commit messages, IEEE, no. 2019 IEEE/ACM 3rd International Workshop on Refactoring (IWoR), 2019. 